\title{
Efecto de dos métodos de secado en los compuestos fenólicos totales, L-DOPA y la actividad antioxidante de Vicia faba $\mathrm{L}$. Effect of two drying methods on the total phenolic compounds, L-DOPA and the antioxidant activity of Vicia faba $\mathrm{L}$.
}

Marly Ortiz López ${ }^{1}$, Adriana Delgado Alvarado ${ }^{1}$, B. Edgar Herrera Cabrera ${ }^{1}$, Ma. De Lourdes Árevalo Galarza ${ }^{2}$ y Ariadna I. Barrera Rodríguez ${ }^{3}$

Palabras clave: antioxidantes; compuestos fenólicos; flor; haba; L-DOPA; plántula Keywords: antioxidants; phenolic compounds; flower; bean; L-DOPA; seedling

Recibido en: 27-05-2019 / Aceptado en: 07-10-2019

\section{Resumen}

La L-DOPA es uno de los metabolitos secundarios presentes en las leguminosas, como Мucuna pruriens y Vicia faba L., su contenido se puede ver afectado por el método de secado, variedad y estado de desarrollo de la planta. El objetivo de este estudio fue analizar el efecto del método de secado por estufa y por liofilización en el contenido de compuestos fenólicos totales (CFT), de LDOPA y de la actividad antioxidante en plántulas y en flor de dos variedades de haba. Dos variedades de haba, Diamante y Zac-22, se utilizaron como material vegetal, que fueron sembradas en condiciones de campo. Se obtuvieron plántulas a los 10, 15 y 20 días después de la emergencia (DDE) y flores durante el período de floración. Los tejidos se sometieron a dos métodos de secado, por estufa de aire forzado a $38{ }^{\circ} \mathrm{C}$ y por liofilización $\left(-80{ }^{\circ} \mathrm{C}\right)$. El contenido de compuestos fenólicos totales (CFT) se determinó por el método de Folin-Ciocalteu y L-DOPA se analizó por HPLC. La actividad antioxidante se determinó frente al radical DPPH (CI50). Los resultados mostraron que el método de secado afectó la concentración de CFT, de L-DOPA y la actividad antioxidante. Las concentraciones más altas de CFT (118.67 $\left.\mathrm{mg} \cdot \mathrm{g}^{-1} \mathrm{MS}\right)$ y de L-DOPA (67.65 $\left.\mathrm{mg} \cdot \mathrm{g}^{-1} \mathrm{MS}\right)$ se detectaron en las muestras procesadas por secado en estufa. Las flores presentaron los contenidos más altos de CFT (142.19 $\left.\mathrm{mg} \cdot \mathrm{g}^{-1} \mathrm{MS}\right)$ y de L-DOPA (82.02 $\mathrm{mg} \cdot \mathrm{g}^{-1}$ MS) respecto a las plántulas. Mientas que en las plántulas a los $10 \mathrm{DDE}$, tuvieron en promedio la concentración mayor de CFT (110.12 $\left.\mathrm{mg} \cdot \mathrm{g}^{-1} \mathrm{MS}\right)$ y de L-DOPA (61.08 $\left.\mathrm{mg} \cdot \mathrm{g}^{-1} \mathrm{MS}\right)$. La actividad antioxidante se afectó particularmente por el método de secado en las plántulas, pero en las flores no mostraron variación. El secado por estufa favoreció la conservación de la actividad

\footnotetext{
${ }^{1}$ Colegio de Postgraduados, Campus Puebla. E-mail: adah@ colpos.mx

${ }^{2}$ Colegio de Postgraduados, Programa en Fruticultura

${ }^{3}$ Universidad Autónoma Chapingo, Preparatoria Agrícola

(C) Universidad De La Salle Bajío (México)
} 
antioxidante de las plántulas $\left(\mathrm{CI}_{50}, 119.93 \mu \mathrm{g} \cdot \mathrm{mL}^{-1}\right)$, respecto al método de secado por liofilización $\left(\mathrm{CI}_{50}, 144.29 \mu \mathrm{g} \cdot \mathrm{mL}^{-1}\right)$.

\begin{abstract}
L-DOPA is one of the secondary metabolites present in legumes, such as Mucuna pruriens and Vicia faba L., its content can be affected by the method of drying, variety and stage of development of the plant. The objective of this study was to analyze the effect of drying method by stove and lyophilization on the content of total phenolic compounds (TPC), L-DOPA and antioxidant activity in seedling and flower of two varieties of broad bean. Two varieties of broad bean, Diamante and Zac-22, were used as plant material, which were sown under field conditions. Seedlings were obtained at 10, 15 and 20 days after the emergence (DAE), and flowers during the flowering period. The tissues were subjected to two drying methods, by forced air oven at $38{ }^{\circ} \mathrm{C}$ and by lyophilization $\left(-80{ }^{\circ} \mathrm{C}\right)$. The content of total phenolic compounds (TPC) was determined by the Folin-Ciocalteu method, and L-DOPA was analyzed by HPLC. The antioxidant activity was determined against the $\mathrm{DPPH}$ radical $\left(\mathrm{IC}_{50}\right)$. The results showed that the drying method affected the concentration of TPC, L-DOPA and antioxidant activity. The highest concentrations of TPC (110.12 $\left.\mathrm{mg} \cdot \mathrm{g}^{-1} \mathrm{DM}\right)$ and L-DOPA $\left(61.08 \mathrm{mg} \cdot \mathrm{g}^{-1} \mathrm{DM}\right)$ were detected in the samples processed by oven drying. Flowers had the highest content of TPC (142.19 $\mathrm{mg}^{\left.-\mathrm{g}^{-1} \mathrm{DM}\right)}$ and L-DOPA (82.02 $\left.\mathrm{mg} \cdot \mathrm{g}^{-1} \mathrm{DM}\right)$ compared to seedlings. While in the seedlings at $10 \mathrm{DAE}$ had on average the highest concentration of TPC $\left(110.12 \mathrm{mg} \cdot \mathrm{g}^{-1} \mathrm{DM}\right)$ and L-DOPA $\left(61.08 \mathrm{mg} \cdot \mathrm{g}^{-1}\right.$ DM). The antioxidant activity was particularly affected by the drying method in the seedlings, but in the flowers, they did not show variation. The drying by stove favored the preservation of the antioxidant activity of the seedlings $\left(\mathrm{IC}_{50}, 119.93 \mu \mathrm{g} \cdot \mathrm{mL}^{-1}\right)$, with respect to the drying method by lyophilization $\left(\mathrm{IC}_{50}, 144.29 \mu \mathrm{g} \cdot \mathrm{mL}^{-1}\right)$.
\end{abstract}

\title{
Introducción
}

Muchas clases de plantas contienen fitoquímicos que se caracterizan por ser componentes bioactivos. Algunos fitoquímicos están presentes en alimentos funcionales de vegetales comestibles. Los fitoquímicos presentes en alimentos funcionales de origen vegetal son de especial interés en la industria farmacéutica para la obtención de fármacos inocuos y efectivos 
para el tratamiento de diversas enfermedades (Foster et al., 2005). La aplicación racional de componentes bioactivos de plantas comestibles como fármacos demanda un conocimiento profundo de sus propiedades farmacológicas para estimar sus efectos terapéuticos y potencial toxicidad (Drago et al., 2006). Dentro de los fitoquímicos se incluyen los terpenos, fitoesteroles, fenoles, lignanos, tioles, tocoferoles y tocotrienoles. Los fenoles protegen a las plantas contra los daños oxidativos y llevan a cabo la misma función en el organismo humano (Chasquibol et al., 2003). La levo-3,4 dihidroxifenilalanina (L-DOPA) que es un aminoácido no-proteico, tiene la estructura de un ácido fenólico (Siqueira-Soares et al., 2013), con el potencial de funcionar como antioxidante para atrapar a los radicales libres generados en el proceso oxidativo (Lewis y ElvinLewis, 2003). Además, L-DOPA es el principal precursor del neurotrasnmisor dopamina, y comúnmente es utilizada en el tratamiento de la enfermedad de Parkinson (Rabey et al., 1992; Ehringer y Hornykiewicz, 1998). Esta enfermedad se caracteriza por una muerte progresiva de neuronas que producen dopamina en los ganglios basales del cerebro (Lang y Lozano, 1998). LDOPA puede cruzar fácilmente la barrera hematoencefálica, para que en las neuronas dopaminérgicas se sintetice la dopamina (Nagatsu y Sawada et al., 2009). Sin embargo, la administración de L-DOPA puede causar efectos secundarios tales como trastornos gastrointestinales, vómitos, náuseas, hipotensión y discinesias (Devi et al., 2004), por lo que debe suministrarse en conjunto con inhibidores de enzimas descarboxilasas periféricas (Jankovic. 2002).

Existen diferentes rutas para la regulación de la biosíntesis de los fenoles como la del acetato-malonato (Dewick, 2002), pentosas fosfato, fenilpropanoides y la del shikimato (Shetty, 1997; Shetty et al., 2001), de esta última ruta se sintetiza L-DOPA. Metabolito que sólo se ha detectado en un número limitados de plantas, y parece estar confinado especialmente en las especies de leguminosas (Andrews y Pridham, 1965), en particular en la familia Fabaceae, donde se ha documentado un contenido relativamente alto (Shetty et al., 2001). Vicia faba L. es una de las principales fuentes de fitoquímicos fenólicos, entre los cuales se encuentra L-DOPA. Cenarruzabeitia et al. (1978) investigaron el contenido de L-DOPA en el haba y establecieron que el contenido aumenta con el período de crecimiento y que su valor más alto se encuentra en las flores. Recientemente Etemadi et al. (2018a) documentaron que en plántulas la concentración más alta de L-DOPA (13.3 $\left.\mathrm{mg}^{-1} \mathrm{~g}^{-1}\right)$ la detectaron a los 15 días después de la germinación y conforme maduraba la planta disminuía el contenido de este metabolito. Por su parte Hu et al. 
(2015) señalaron que en flores, el contenido de L-DOPA estuvo en un rango entre 27.8 a 63.5 $\mathrm{mg} \cdot \mathrm{g}^{-1}$ de peso seco, por lo que mencionan que las flores llegan a tener e contenido más alto de L-DOPA dentro de la planta de Vicia faba L.

La L-DOPA de fuentes naturales reduce los efectos secundarios en los pacientes de la enfermedad de Parkinson, además de tener un costo más bajo y retardar la progresión de la enfermedad en relación con la L-DOPA sintetizada químicamente (Patil et al., 2013). Con todo y esto se requeriría del inhibidor de la DOPA descarboxilasa para evitar que se convierta rápidamente a dopamina fuera del cerebro, y permitir que aumente su biodisponibilidad a nivel central (Menéndez-González et al., 2016). Debido a que L-DOPA puede atravesar la barrera hematoencefálica mientras que la dopamina no. Algunos estudios clínicos en el manejo de la enfermedad de Parkinson, han demostrado que el consumo de leguminosas que producen LDOPA, como extractos de semillas de Mucuna pruriens (Hussian y Manyam, 1997) y de vainas y plántulas de Vicia faba L. (Vered et al., 1997; Apaydin et al. 2000; Devi et al., 2004) tienen un buen potencial. Los resultados de esos estudios han evidenciado incrementos en los niveles de LDOPA en la sangre con una mejora notable en el rendimiento motor de los pacientes sin efectos secundarios.

Un inconveniente de las especies vegetales que contienen L-DOPA en los tejidos frescos como Vicia faba L., es que no están disponibles en todas las épocas del año ni en cualquier localidad. Una alternativa promisoria es utilizar la planta o partes de la planta procesada por diferentes métodos, como tejidos congelados o tejidos pulverizados secos (Etemadi et al., 2018a). Si bien algunos reportes han documentado el impacto negativo que pueden tener los métodos de procesamiento en la concentración de L-DOPA en semillas de Mucuna pruriens (Echeverria y Bressani, 2006) y de Vicia faba L. (Cardador-Martínez et al., 2012), no está completamente descrito en otros tejidos de la planta de haba, a qué nivel influyen métodos de procesamiento como el secado por estufa a baja temperatura o bien el secado por liofilización. El objetivo de este estudio fue determinar el efecto de dos métodos de secado en el contenido de compuestos fenólicos totales, de L-DOPA y de la actividad antioxidante en plántulas y flores de dos variedades de haba con diferente precocidad cultivadas en condiciones de campo. 


\section{Materiales y métodos}

\section{Material vegetal}

Se utilizaron semillas de haba de diferente precocidad, la variedad Zac-22 (45 - 50 días a la floración), y la variedad Diamante (65 días a la floración). El experimento se realizó en campo abierto, en los meses de marzo a junio de 2018, en la localidad San Agustín Calvario, Cholula, Puebla. Las características físico-químicas del suelo fueron de textura arena migajonosa, ligeramente alcalina $(\mathrm{pH}=7.1)$ y con $0.75 \%$ de materia orgánica; rico en fósforo $\left(62 \mathrm{mg} \cdot \mathrm{kg}^{-1}\right)$ y hierro $\left(60 \mathrm{mg} \cdot \mathrm{kg}^{-1}\right)$, con concentraciones adecuadas de $\mathrm{Na}\left(0.34 \mathrm{cmol} \cdot \mathrm{kg}^{-1}\right), \mathrm{Cu}\left(1.28 \mathrm{mg} \cdot \mathrm{kg}^{-1}\right)$, Mn $\left(1.18 \mathrm{mg} \cdot \mathrm{kg}^{-1}\right)$ y Zn $\left(2.3 \mathrm{mg} \cdot \mathrm{kg}^{-1}\right)$, pero pobre en $\mathrm{K}\left(0.28 \mathrm{cmol} \cdot \mathrm{kg}^{-1}\right), \mathrm{Ca}\left(3.75 \mathrm{cmol} \cdot \mathrm{kg}^{-1}\right), \mathrm{Mg}$ $\left(1.75 \mathrm{cmol} \cdot \mathrm{kg}^{-1}\right)$ y CIC $\left(7 \mathrm{cmol} \cdot \mathrm{kg}^{-1}\right)$. La temperatura promedio durante el experimento se mantuvo en $20^{\circ} \mathrm{C}$, mientras que la máxima estuvo en un rango entre $38-45^{\circ} \mathrm{C}$, y la temperatura mínima en $7^{\circ} \mathrm{C}$. La humedad relativa (HR) presentó un promedio de $60 \%$, pero en los meses de mayo a junio fue de alrededor de $80 \%$. Se realizó un riego a la siembra. Las semillas de las dos variedades fueron sembrados en parcelas de $4.5 \mathrm{~m}^{2}(3 \times 1.5 \mathrm{~m})$ a una profundidad de $5 \mathrm{~cm}$, cada parcela estuvo conformada por tres surcos, la distancia entre ellos fue de $60 \mathrm{~cm}$, con una distancia entre plantas de $30 \mathrm{~cm}$. Cada parcela fue una repetición, estableciéndose cuatro repeticiones de cada variedad.

Cada tercer día se visitó el terreno de cultivo entre las 7:00 y 8:30 am, y en el momento de la emergencia de las plántulas, cuando se observó el epicótilo (tallo) expuesto por arriba del nivel del suelo, cada plántula se marcó con una cinta de estambre de diferente color, para identificar la edad de las plántulas. A los 10, 15 y 20 días después de emergencia (DDE) se recolectaron aproximadamente 25 plántulas de haba de cada edad.

\section{Proceso de secado}

Procesamiento por horno de convección. De cada variedad, 10 plántulas de cada una de las edades se introdujeron en bolsas de papel de estraza, y se sometieron a deshidratación a $38 \pm 2{ }^{\circ} \mathrm{C}$ por 12 - $14 \mathrm{~h}$ en el horno con flujo de aire forzado (Shel Lab, Modelo 1370FX, E.U.A.), hasta obtener un porcentaje de humedad en los tejidos de $6-7 \%$.

Procesamiento por liofilización. Inmediatamente después del corte las plántulas de las diferentes edades y del registro del peso fresco, se partieron en trozos de aproximadamente $1 \mathrm{~cm}$, se colocaron en bolsas de tul debidamente etiquetadas y se congelaron a $-70{ }^{\circ} \mathrm{C}$, por $48 \mathrm{~h}$. 
Ortiz López et al.

Posteriormente las muestras previamente congeladas en las bolsas se introdujeron a una liofilizadora (Labconco, catalogo 7400040, E.U.A), con un Sistema de Liofilización FreeZone® Triad, y se procesaron a una presión de $0.22 \mathrm{mBa}$, con una temperatura en el colector de $-80{ }^{\circ} \mathrm{C}$, por un periodo de aproximadamente $18 \mathrm{~h}$ hasta obtener tejidos con un porcentaje de humedad entre $6.5-7 \%$.

Las plantas deshidratas por ambos procesos se molieron en un molino (Krups, Gx4100, E.U.A), el residuo molido se pasó a un mortero para un molido más fino de la muestra, posteriormente, se tamizó a un tamaño de partícula $420 \mu \mathrm{m}$ y luego se conservaron en frascos ámbar a temperatura ambiente $\left(22 \pm 2{ }^{\circ} \mathrm{C}\right)$.

\section{Determinación de compuestos fenólicos totales}

Se utilizó el micro-método de Folin-Ciocalteu propuesto por Singleton y Rossi (1965). Se pesó 5 $\mathrm{mg}$ de tejido vegetal y se disolvió en $5 \mathrm{~mL}$ de metanol, posteriormente las muestras se colocaron en un baño ultrasónico (Ultrasonic Cleaner AS5150B, China) por 30 min y después se centrifugaron a 5488 x $g$ por 5 min. Los sobrenadantes se utilizaron como extractos y se mantuvieron en refrigeración a $4{ }^{\circ} \mathrm{C}$ hasta su uso. Para los ensayos se utilizó $25 \mu \mathrm{L}$ del sobrenadante (o de estándar ácido gálico), se añadió $75 \mu \mathrm{L}$ de agua desionizada. En seguida se agregó a la mezcla $25 \mu \mathrm{L}$ de reactivo de fenol de Folin-Ciocalteu (Sigma Aldrich, No. Cat. 47641, Suiza) (1:1 en agua desionizada), se agitó por 10 s a $72 \mathrm{rpm}$ en parrilla de agitación (Thermo Scientific, SP131015, Malasia). Se dejó reposar por 6 min a temperatura ambiente (22 \pm $\left.2{ }^{\circ} \mathrm{C}\right)$ en la oscuridad. Se añadió $100 \mu \mathrm{L}$ de carbonato de sodio $\left(\mathrm{Na}_{2} \mathrm{CO}_{3}\right.$, J.T. Baker, numero de catálogo: 3604, E.U.A) al 7.5\% (p/v) (disuelto en agua destilada). Se agitó por $10 \mathrm{~s}$ a $72 \mathrm{rpm}$. Se dejó reposar a temperatura ambiente por $60 \mathrm{~min}$ en la oscuridad. La lectura de absorbancia se registró a $725 \mathrm{~nm}$ en un espectrofotómetro de microplaca (Thermo Scientific Varioskan Flash, Reino Unido).

\section{Determinación de L-DOPA}

La cuantificación de L-DOPA se realizó por triplicado en tejidos de plántulas de 10, 15 y 20 DDE y en flores por medio del método de St-Laurent et al. (2000) con algunas adecuaciones. Se pesó $35 \mathrm{mg}$ de las muestras de las plantas y se agregó $7.5 \mathrm{~mL}$ de la solución extractora agua: acetonitrilo (85:15) (v/v) a pH 5 y se conservaron en frascos ámbar en refrigeración a $4{ }^{\circ} \mathrm{C}$ por 
toda la noche. Al día siguiente, los frascos con las muestras se mantuvieron en agitación por 30 min a 72 rpm en parrilla de agitación (Thermo Scientific, SP131015, Malasia). Posteriormente la mezcla se centrifugó a 2800 x $g$ por 10 min a una temperatura de $10{ }^{\circ} \mathrm{C}$. Del sobrenadante de cada muestra se realizó una dilución 1:10 en fase móvil, que fue preparada con 98\% de ácido acético y $2 \%$ de metanol. El extracto se filtró con acrodiscos de $30 \mathrm{~mm}$ con una membrana de nylon de poro de $0.45 \mu \mathrm{m}$ (Titan 2, Thomas Scientific, E.U.A.), el filtrado se colocó en viales ámbar de $2 \mathrm{~mL}$ para realizar el análisis. Para el análisis se usó un equipo HPLC (Perkin Elmer, 200a, E.U.A) con un detector de arreglo de diodos y una columna SuperC18 (5 $\mu \mathrm{m}$ tamaño de partícula) (250 x $4.6 \mathrm{~mm}$ di) (ACE Ultra Core, ACE-121-2546, Reino Unido). La fase móvil consistió de $0.1 \%$ de ácido acético glacial (98\%) y $2 \%$ de metanol. El volumen de muestra fue de $10 \mu \mathrm{L}$, el flujo fue isocrático $\left(1 \mathrm{~mL} \cdot \mathrm{min}^{-1}\right)$ y la detección fue a una longitud de onda de $283 \mathrm{~nm}$, con una temperatura de análisis de $25^{\circ} \mathrm{C}$.

\section{Actividad antioxidante}

La actividad antioxidante in vitro frente al radical DPPH se analizó con base en el método BrandWilliams et al. (1995) con algunas modificaciones para micrométodo. En el ensayo se utilizaron $50 \mu \mathrm{L}$ del mismo extracto usado para el análisis de compuestos fenólicos totales, y se mezcló con $200 \mu \mathrm{L}$ de DPPH 0.1 mM (1,1-difenil-2-picrilhidrazil; Sigma-Aldrich, No Cat. D9132), después la mezcla se agitó por $10 \mathrm{~s}$ a velocidad media. La absorbancia se determinó a una longitud de onda de $521 \mathrm{~nm}$, cada minuto por un periodo de $30 \mathrm{~min}$, en el blanco (metanol) ( $\mathrm{A}_{0}$ ) y en las muestras $\left(\mathrm{A}_{1}\right)$, en un espectrofotómetro de microplaca (Thermo Scientific, Varioskan Flash, Reino Unido).

La actividad antioxidante se expresó como porcentaje de inhibición, lo cual corresponde a la cantidad de radical DPPH neutralizado por el extracto a una determinada concentración, de acuerdo a la siguiente ecuación: \% Inhibición=\% $I=\left[A-A_{l} / A_{0}\right](100)$.

Se calculó el $\mathrm{CI}_{50}$, que es la concentración media inhibitoria que causa la reducción del $50 \%$ de color y concentración del DPPH. Para obtener el $\mathrm{CI}_{50}$ se realizaron diluciones del extracto de metanol a diferentes concentraciones de los tejidos analizados de las plántulas de haba: 40, 60, 80, 100, 200 y $400 \mu \mathrm{g} \cdot \mathrm{mL}^{-1}$. El cálculo se realizó mediante una regresión lineal de al menos cuatro puntos de los resultados del porcentaje de inhibición del extracto de las 
diferentes diluciones. Por lo que, a mayor actividad antioxidante, el valor de $\mathrm{CI}_{50}$ será menor (Kedare y Singh, 2011).

\section{Análisis estadístico}

Los datos por proceso de secado se analizaron mediante un modelo equivalente al diseño de bloques incompletos al azar, con cuatro repeticiones para cada variedad, desbalanceado (PROC GLM, SAS, 2002). Las variables de contenido de compuestos fenólicos totales, de L-DOPA y la actividad antioxidante fueron sometidas a un análisis de varianza, las cuales se evaluaron en tres diferentes fechas de corte (10, 15 y 20 DDE), con tres repeticiones por cada fecha de corte. La comparación de medias para cada fuente de variación se realizó mediante la prueba de Tukey.

\section{Resultados y discusión}

Efecto de variedad, tejido, edad y secado en el contenido de compuestos fenólicos totales, L-

\section{DOPA y actividad antioxidante}

Los resultados indicaron un efecto significativo del tejido, edad y método de secado de la plántula $(\mathrm{P}<0.0001)$ sobre compuestos fenólicos totales y L-DOPA. Mientras que la variedad sólo mostro un efecto significativo en la actividad antioxidante (Cuadro 1). El coeficiente de variación tuvo una amplitud entre 20.25 y $29.76 \%$.

Cuadro 1. Medias, coeficientes de variación y cuadrados medios de las características químicas del cultivo de haba de dos variedades conservadas por dos tipos de secado.

\begin{tabular}{|c|c|c|c|c|c|c|c|}
\hline \multirow[t]{2}{*}{ Variable } & \multirow[t]{2}{*}{ Media } & \multirow{2}{*}{$\begin{array}{l}\text { CV } \\
(\%)\end{array}$} & \multicolumn{3}{|c|}{ Cuadrados de la media } & & \multirow[t]{2}{*}{ Error } \\
\hline & & & Variedad & Tejido & Edad & $\begin{array}{l}\text { Método de } \\
\text { proceso }\end{array}$ & \\
\hline $\begin{array}{c}\text { CFT } \\
\left(\mathrm{mg} \cdot \mathrm{g}^{-1} \mathrm{MS}\right)\end{array}$ & 106.32 & 20.25 & $32.93 \mathrm{~ns}$ & $64291.30 * * *$ & $7794.96 * * *$ & $73613.35 * * *$ & 461.92 \\
\hline $\begin{array}{c}\text { L-DOPA } \\
\left(\mathrm{mg} \cdot \mathrm{g}^{-1} \mathrm{MS}\right)\end{array}$ & 58.95 & 23.19 & $189.21 \mathrm{~ns}$ & $8112.86 * * *$ & $982.26 * * *$ & $3632.46 * * *$ & 181.86 \\
\hline $\begin{array}{c}\mathrm{DPPH}\left(\mathrm{CI}_{50}\right) \\
\left(\mu \mathrm{g} \cdot \mathrm{mL}^{-1}\right)\end{array}$ & 132.44 & 29.76 & $50528.06 * * *$ & $30152.76^{* * *}$ & $6757.36^{* * *}$ & $22217.70 * * *$ & 1563.17 \\
\hline
\end{tabular}

CFT = Compuestos fenólicos totales; $\mathbf{C V = C o e f i c i e n t e ~ d e ~ V a r i a c i o ́ n . ~ D D E = D i ́ a s ~}$

Después de Emergencia; $* * *=\mathrm{P} \leq 0.0001 ; \mathbf{n s}=$ no significativo. 
Aunque la literatura documenta que la concentración de L-DOPA varía entre cultivares o variedades de haba (Etemadi et al., 2014; Hu et al., 2015), las dos variedades de haba utilizadas en este trabajo no mostraron diferencias en la concentración de CFT ni en la de L-DOPA (Cuadro 2). La actividad antioxidante frente al radical DPPH mostró una actividad mayor en la variedad Diamante $\left(\mathrm{CI}_{50}: 114.99 \mu \mathrm{g} \cdot \mathrm{mL}^{-1}\right)$ (Cuadro 2). Por tejido, la flor tuvo una concentración mayor de CFT y de L-DOPA, y también una actividad antioxidante mayor. La edad de las plántulas afectó la concentración de los metabolitos. Se detectó que las plántulas más jóvenes de 10 DDE mostraron la concentración más alta de CFT (110.12 $\left.\mathrm{mg} \cdot \mathrm{g}^{-1} \mathrm{MS}\right)$ y de L-DOPA $\left(61.08 \mathrm{mg} \cdot \mathrm{g}^{-1}\right.$ MS), y también las plántulas de 10 y 15 DDE presentaron una actividad inhibitoria mayor que las plántulas de 20 DDE (Cuadro 2).

Cuadro 2. Promedio de la concentración de compuestos fenólicos totales (CFT), LDOPA y actividad antioxidante $\left(\mathrm{CI}_{50}\right)$ frente al radical DPPH evaluadas en dos variedades de Vicia faba $\mathrm{L}$.

\begin{tabular}{|c|c|c|c|}
\hline Factor & 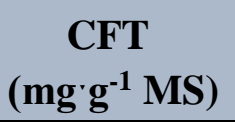 & $\begin{array}{c}\text { L-DOPA } \\
\left(\mathrm{mg}^{\prime} \mathrm{g}^{-1} \mathrm{MS}\right)\end{array}$ & $\begin{array}{c}\mathrm{DPPH}\left(\mathrm{CI}_{50}\right) \\
\left(\mu \mathrm{g} \cdot \mathrm{mL}^{-1}\right)\end{array}$ \\
\hline \multicolumn{4}{|l|}{ Variedad } \\
\hline Diamante & $106.36^{\mathrm{a}}$ & $60.09^{\mathrm{a}}$ & $114.99^{\mathrm{a}}$ \\
\hline Zac-22 & $105.79^{\mathrm{a}}$ & $56.08^{\mathrm{a}}$ & $152.23^{\mathrm{b}}$ \\
\hline DMS & 4.20 & 7.93 & 12.94 \\
\hline \multicolumn{4}{|l|}{ Tejido } \\
\hline Plántula & $101.69^{\mathrm{b}}$ & $50.83^{\mathrm{b}}$ & $137.44^{\mathrm{b}}$ \\
\hline Flor & $142.19^{\mathrm{a}}$ & $82.02^{\mathrm{a}}$ & $89.48^{\mathrm{a}}$ \\
\hline DMS & 6.74 & 9.36 & 21.96 \\
\hline \multicolumn{4}{|l|}{ Edad } \\
\hline $10 \mathrm{DDE}$ & $110.12^{\mathrm{a}}$ & $61.08^{\mathrm{a}}$ & $126.93^{\mathrm{a}}$ \\
\hline $15 \mathrm{DDE}$ & $100.20^{\mathrm{b}}$ & $49.04^{\mathrm{b}}$ & $136.01^{\mathrm{a}}$ \\
\hline $20 \mathrm{DDE}$ & $94.16^{\mathrm{b}}$ & $42.78^{\mathrm{b}}$ & $151.81^{\mathrm{b}}$ \\
\hline DMS & 6.27 & 11.99 & 20.19 \\
\hline \multicolumn{4}{|l|}{ Método de secado } \\
\hline Estufa & $118.67^{\mathrm{a}}$ & $67.65^{\mathrm{a}}$ & $119.93^{\mathrm{a}}$ \\
\hline Liofilizado & $92.21^{\mathrm{b}}$ & $50.25^{\mathrm{b}}$ & $144.29^{\mathrm{b}}$ \\
\hline DMS & 3.13 & 4.62 & 11.79 \\
\hline
\end{tabular}

DDE= Días Después de la Emergencia. Letras diferentes por factor en cada columna indican diferencia significativa Tukey $(\mathrm{P} \leq 0.05)$; DMS = Diferencia mínima significativa. 
El contenido mayor de compuestos fenólicos totales en etapas tempranas de la planta se debe a la necesidad de activar un mecanismo de defensa hacia el medio que los rodea. Al respecto se sabe que las condiciones del ambiente y el estrés ambiental afecta la acumulación de los metabolitos en las plantas (Afshar et al., 2015). Según Chon (2013) los compuestos fenólicos son metabolitos secundarios sintetizados durante el desarrollo normal y responden a condiciones de estrés y también se encuentran en diferentes partes de la planta.

Las plántulas secadas en estufa tuvieron una concentración significativamente mayor tanto para los CFT (118.67 $\mathrm{mg} \cdot \mathrm{g}^{-1} \mathrm{MS}$ ) como para L-DOPA (67.65 $\mathrm{mg} \cdot \mathrm{g}^{-1} \mathrm{MS}$ ) (Cuadro 2). El secado de tejidos vegetales con temperaturas mayores a $60{ }^{\circ} \mathrm{C}$ puede inducir la condensación oxidativa o degradación de compuestos termolábiles, mientras que la liofilización crea cristales de hielo en la planta, que rompen la estructura celular y permiten mejor el acceso y la extracción de los solventes (Asami et al., 2003). Los resultados de nuestro estudio coinciden con la teoría mencionada, ya que el secado en estufa a una temperatura de $38{ }^{\circ} \mathrm{C}$ fue más efectivo para la conservación de L-DOPA. De acuerdo con Mediani et al. (2014) el método de secado en algunas circunstancias puede ser beneficioso para proteger algunos compuestos y no reducir su calidad siempre y cuando se mantengan condiciones adecuadas de temperaturas menores a $60{ }^{\circ} \mathrm{C}$. Lo cual corresponde con nuestros resultados en la temperatura utilizada, y con un mayor contenido de L-DOPA.

\section{Comparación de niveles y de rendimiento de los compuestos fenólicos totales y L-DOPA por variedad y por método de secado}

El rendimiento de los CFT y de L-DOPA se consideró como el producto de la concentración de cada uno de ellos con base en la materia seca de las plántulas. El patrón de acumulación de la concentración y de rendimiento de CFT por variedad presentó una tendencia diferente en relación con el método de procesamiento de secado de las plántulas de haba. En el método de secado en estufa las plántulas de edad más joven (10 DDE) tanto de la variedad Diamante $\left(125.94 \mathrm{mg} \cdot \mathrm{g}^{-1}\right.$ MS) como la variedad Zac-22 (121.42 $\left.\mathrm{mg} \cdot \mathrm{g}^{-1} \mathrm{MS}\right)$ presentaron los contenidos más altos y tendieron a disminuir en relación con las de mayor edad (20 DDE), contrario a la expresión con base en rendimiento $\left(\mathrm{mg} \cdot\right.$ plantula $\left.^{-1}\right)$ que mostró un valor mayor o igual a los $20 \mathrm{DDE}$, donde la variedad Diamante alcanzó un rendimiento de $119.02 \mathrm{mg} \cdot$ plantula $^{-1}$ y de $127.30 \mathrm{mg} \cdot$ plantula $^{-1}$ en la variedad Zac-22 (Fig. 1). En el procesamiento por liofilización, en la variedad precoz (Zac-22) 
la tendencia en la concentración de CFT fue similar a disminuir de los 10 a los 20 DDE, aunque el rendimiento por plántula (58.06 mg. plantula ${ }^{-1}$ ) a los 20 DDE fue menor. Sin embargo, en la variedad Diamante que es de floración tardía, la concentración en las plántulas $\left(93.53 \mathrm{mg} \cdot \mathrm{g}^{-1}\right.$ MS) de 20 DDE se mantuvo similar al rendimiento por plántula (90.59 mg.plantula $\left.{ }^{-1}\right)$ (Fig. 1).

Se sabe que la inestabilidad y sensibilidad de los compuestos fenólicos en plantas suelen estar relacionado a ciertos tratamientos de secado (Lim y Murtijaya 2007). En general nuestros resultados mostraron que el método de secado en estufa a $38^{\circ} \mathrm{C}$ presentó los contenidos más altos de CFT en relación a las muestras secadas por liofilización a $-80{ }^{\circ} \mathrm{C}$. En contraste, en la especie Cosmos caudatus de la familia Astereacea y de amplia importancia en la medicina tradicional, se menciona que el secado por liofilización con congelamiento previo a $-20{ }^{\circ} \mathrm{C}$, favoreció una mayor conservación de CFT en relación con el secado por estufa a $44.5^{\circ} \mathrm{C}$ (Mediani et al., 2014) en el que se redujo considerablemente la concentración de estos compuestos por el efecto del calor.

Probablemente los resultados de nuestro estudio difieren, debido a que utilizamos una temperatura de secado en estufa de aire forzado más baja $\left(38^{\circ} \mathrm{C}\right)$, que la utilizada en el trabajo de C. caudatus, y la temperatura de congelamiento previo de las muestras y del colector durante la liofilización, fue mayor $\left(-80{ }^{\circ} \mathrm{C}\right)$. Además, como mencionan Petkovsek et al. (2010) que la acumulación de compuestos fenólicos puede ser influenciada por las condiciones de cultivo de la especie, condiciones climáticas, estación de cultivo, entre otras.

La literatura reporta que el procesamiento de los tejidos de leguminosas afecta de forma negativa la concentración de L-DOPA, como en las semillas de Mucuna pruriens, donde la concentración disminuye por el proceso de cocimiento a alta temperatura y el uso de soluciones alcalinas (Echeverría y Bressani, 2006). Para esta misma especie también señalan que la cocción de las semillas afecta la concentración de L-DOPA pero no el tostado (Dahouda et al., 2009). Mientras que en los cotiledones de semillas maduras de Vicia faba L., la L-DOPA se removió completamente por tratamientos térmicos de hervido y tostado a $120{ }^{\circ} \mathrm{C}$ (Cardador-Martínez et al., 2012), lo que apunta que es un compuesto termolábil.

La revisión realizada recientemente por Etemadi et al. (2019) sobre aspectos agronómicos, de valor nutricional y aplicaciones medicinales de Vicia faba L., refiere que "la distribución de L-DOPA no es uniforme en las estructuras de la planta de haba, y que los diferentes órganos acumulan L-DOPA en diferentes proporciones”. Etemadi et al. (2018b) señala 
que el pico de concentración de L-DOPA en los órganos de Vicia faba L. se alcanza en estados tempranos del desarrollo, como se observó en este estudio, donde ambas variedades de haba acumularon la concentración mayor a los 10 DDE, pero a esta misma edad expresado con base en la biomasa por plántula se tuvieron los valores más bajos (Fig. 2). Debido a que en Vicia faba, el rendimiento de L-DOPA es el producto de la concentración y biomasa de la planta (Etemadi et al., 2018b), de tal forma que a medida que la planta se desarrolla se puede obtener un rendimiento mayor con base en la biomasa. Por ejemplo, las muestras procesadas por secado en estufa a los 10 DDE de la variedad Diamante tuvieron la concentración más alta $\left(65.26 \mathrm{mg} \cdot \mathrm{g}^{-1}\right.$ MS) y similar a la variedad Zac-22 (66.82 $\left.\mathrm{mg} \cdot \mathrm{g}^{-1} \mathrm{MS}\right)$. Sin embargo, a esta misma edad, el contenido de L-DOPA expresado por plántula ${ }^{-1}$ tuvo los valores más bajos, $34.57 \mathrm{mg} \cdot$ plántula $^{-1}$ en la variedad Diamante y 37. 68 mg. plántula $^{-1}$ en Zac-22 (Fig. 2).
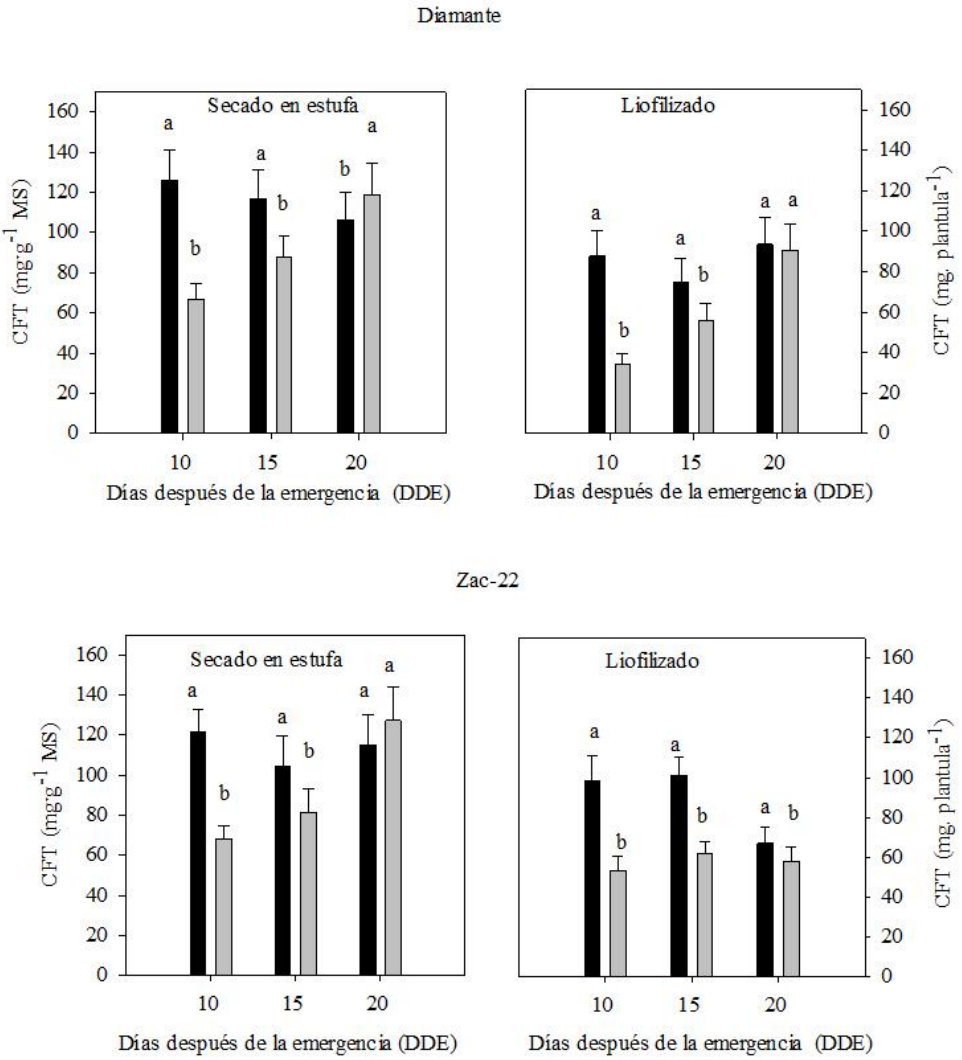

Fig. 1. Contenido de compuestos fenólicos totales (CFT) a los 10, 15 y 20 días después de emergencia (DDE), en las variedades de haba, Diamante y Zac-22, expresados en mg por gramo de materia seca (MS) ( $\square$ ) y por plántula ( $\square$ ). Cada punto es la media de tres repeticiones \pm desviación estándar. Barras con letras diferentes por edad son estadísticamente diferentes (Tukey $\mathrm{P} \leq 0.05$ ). 
Aunque en general el secado por liofilización mostró una tendencia similar de acumulación de LDOPA en las diferentes edades de las plántulas como en el secado por estufa, en la variedad Diamante a los 20 DDE se obtuvieron valores similares expresados tanto en concentración (53.30 $\left.\mathrm{mg} \cdot \mathrm{g}^{-1}\right)$ como por plántula (59.79 $\mathrm{mg} \cdot$ plántula $\left.^{-1}\right)$ (Fig. 2). Inclusive en la variedad Zac-22 el contenido fue mayor expresado en concentración $\left(50.24 \mathrm{mg} \cdot \mathrm{g}^{-1}\right)$ que por plántula $(55.52$ $\mathrm{mg} \cdot$ plántula $\left.^{-1}\right)$. Lo que sugiere que el método de liofilización afectó significativamente el contenido de L-DOPA, probablemente por la baja temperatura de congelamiento $\left(-80^{\circ} \mathrm{C}\right)$ previo al proceso y durante la liofilización.

Es notable destacar que bajo las condiciones de este estudio, de plantas sembradas en suelo y en campo abierto con valores promedio de radiación fotosintéticamente activa de 788 $\mu \mathrm{mol} \mathrm{m} \mathrm{m}^{-2} \mathrm{~s}^{-1}, 20{ }^{\circ} \mathrm{C}$ de temperatura, y $60 \%$ de humedad relativa durante el periodo de cultivo de las plantas, las concentraciones más altas de L-DOPA se obtuvieron a los 10 DDE $\left(110.12 \mathrm{mg} \cdot \mathrm{g}^{-1}\right.$ MS) en promedio de ambas variedades. En contraste en plantas de Vicia faba L. cultivadas en macetas de plástico con perlita y crecidas bajo iluminación continua de luz blanca fluorescente (1500 $\mu \mathrm{mol} \mathrm{m} \mathrm{m}^{-2}$ ), las plántulas de 10-12 de edad presentaron contenidos de L-DOPA de 8.70$40.01 \mathrm{mg} \cdot \mathrm{g}^{-1}$ de MS y los valores más bajos los detectaron en las semillas (Kirakosyan et al., 2004), por lo que estos autores explican que las rutas para la biosíntesis de L-DOPA se activan después de la germinación y durante el desarrollo de la planta.

\section{Actividad antioxidante}

Diferentes compuestos como vitaminas, polifenoles, carotenoides, terpenoides, y minerales traza pueden actuar en sinergia para conferir la capacidad antioxidante a las plantas (Pérez-Jiménez et al., 2008). En particular, los compuestos fenólicos son fitoquímicos con una alta relevancia por sus propiedades que promueven la salud. Recientemente Turco et al. (2016) realizaron una revisión sobre los beneficios para la salud de los polifenoles de Vicia faba L., y señalan que estos compuestos en el haba podrían estar implicados en la protección contra el desarrollo de enfermedades humanas. En este trabajo se calculó la concentración de extracto necesaria para disminuir la concentración inicial de DPPH en $50 \%\left(\mathrm{CI}_{50}\right)$ bajo las condiciones experimentales especificadas, tanto en plántulas de diferente edad como en flores de dos variedades de haba bajo dos métodos de secado. 


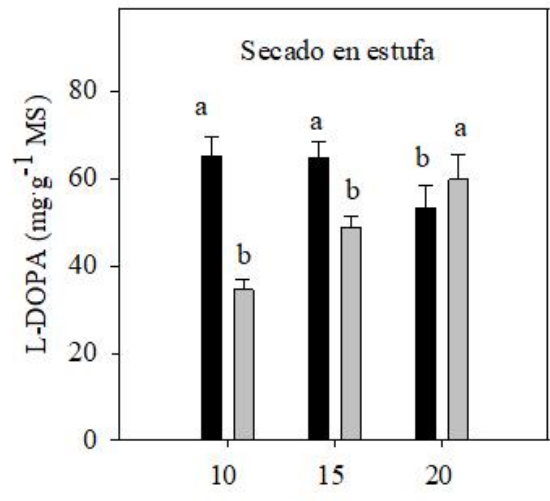

Días después de la emergencia (DDE)

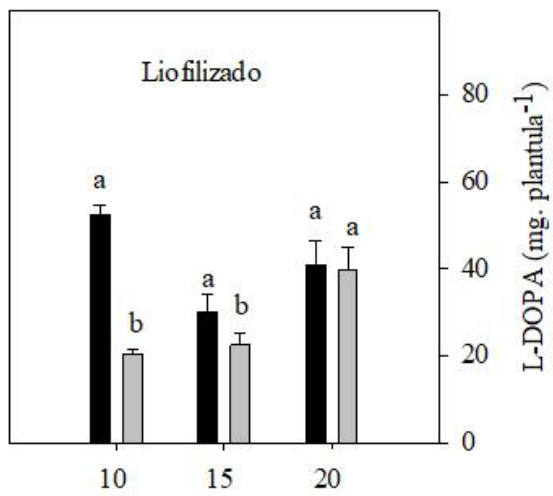

Días después de la emergencia (DDE)

Zac- 22
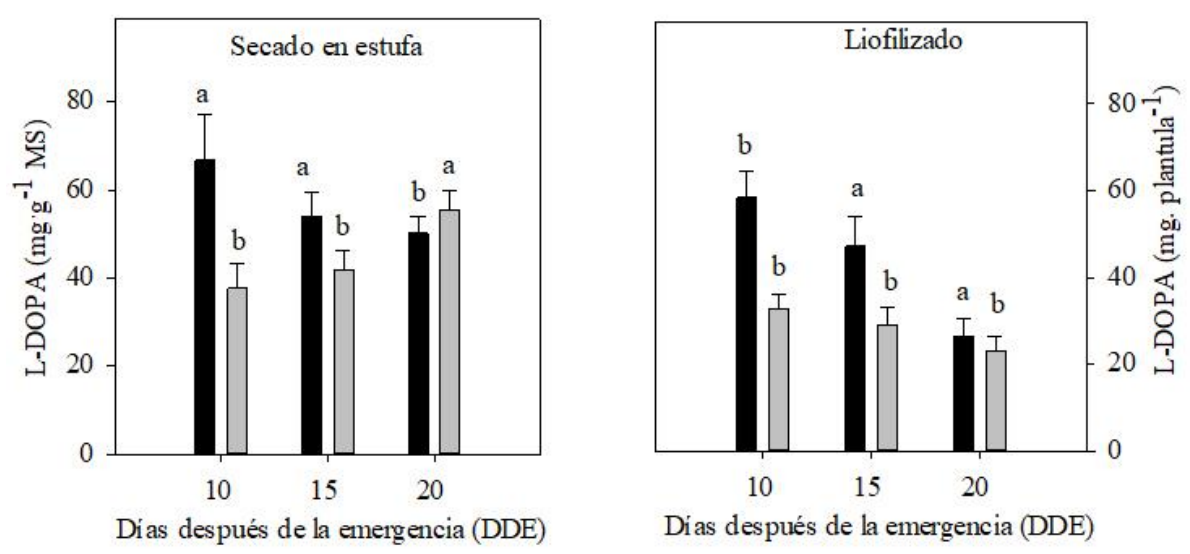

Fig. 2. Contenido de L-DOPA expresado en mg por gramo de materia seca ( $\square$ ) y rendimiento por plántula $(\square)$ de las variedades de Vicia faba L., Diamante y Zac-22 a los 10, 15 y 20 días después de emergencia. Cada punto es la media de tres repeticiones \pm desviación estándar. Barras con letras diferentes por edad son estadísticamente diferentes (Tukey $\mathrm{P} \leq 0.05$ ).

Los resultados obtenidos en la determinación de la actividad antioxidante $\mathrm{CI}_{50}$ mostraron diferencia significativa $(\mathrm{P}<0.05)$ por variedad, entre métodos de secado, dentro de tejidos (Cuadro 3). En la variedad Diamante el secado afectó de manera diferente en los tejidos, los extractos de las plántulas de 10 y 15 DDE tuvieron una actividad inhibidora del radical DPPH significativamente mayor en el método de secado por estufa respecto al liofilizado. En contraste, las plántulas de 20 DDE y las flores liofilizadas mostraron una actividad antioxidante significativamente mayor respecto al secado por estufa (Cuadro 3). En la variedad Zac-22, tanto 
en plántula como en flor, el método de secado por estufa mantuvo la actividad inhibitoria mayor respecto a los tejidos liofilizados (Cuadro 3).

Cuadro 3. Actividad antioxidante $\left(\mathrm{CI}_{50}\right)$ frente al radical DDPH de plántulas de 10 , 15 y 20 días después de la emergencia (DDE) y flor secadas en estufa y por liofilización de dos variedades de Vicia faba $\mathrm{L}$.

\begin{tabular}{|c|c|c|c|c|c|}
\hline \multirow{3}{*}{\multicolumn{2}{|c|}{ Tejido }} & \multicolumn{4}{|c|}{$\mathrm{CI}_{50}\left(\boldsymbol{\mu g} \cdot \mathrm{mL}^{-1}\right)$} \\
\hline & & \multicolumn{2}{|c|}{ Diamante } & \multicolumn{2}{|c|}{ Zac-22 } \\
\hline & & Estufa & Liofilizado & Estufa & Liofilizado \\
\hline \multicolumn{6}{|c|}{ Plántula (DDE) } \\
\hline & 10 & $108.40^{\mathrm{a}}$ & $136.56^{\mathrm{b}}$ & $123.33^{\mathrm{a}}$ & $138.05^{\mathrm{b}}$ \\
\hline & 15 & $121.07^{\mathrm{a}}$ & $134.07^{\mathrm{b}}$ & $140.21^{\mathrm{a}}$ & $149.82^{b}$ \\
\hline & 20 & $121.04^{\mathrm{b}}$ & $85.35^{\mathrm{a}}$ & $130.57^{\mathrm{a}}$ & $259.89^{\mathrm{b}}$ \\
\hline Flor & & $96.18^{\mathrm{b}}$ & $74.95^{\mathrm{a}}$ & $70.28^{\mathrm{a}}$ & $115.28^{\mathrm{b}}$ \\
\hline
\end{tabular}

Letras diferentes en cada fila por variedad entre métodos de secado indican diferencia significativa Tukey $(\mathrm{P} \leq 0.05)$.

Algunos autores mencionan que los tratamientos de calentamiento pueden desactivar algunas enzimas y también degradar algunos fitoquímicos causando un contenido bajo de metabolitos, específicamente en los antioxidantes. Debido a que algunos procesos de secados pueden beneficiar o no a la actividad natural de los antioxidantes, ya que muchos de ellos suelen ser muy inestables a altas temperaturas (Lim y Murtijaya 2007). En este estudio el método de secado y el estado fenológico influyeron en la actividad antioxidante, en general, el método de secado en estufa y las flores mantuvieron una alta capacidad de captación de radicales libres del DPPH $\left(\mathrm{CI}_{50}\right)$.

Existe una clasificación de la capacidad antioxidante de los extractos vegetales en relación a la concentración del valor de $\mathrm{CI}_{50}$ (Ramos et al., 2003). Así extractos con alto potencial antioxidante se refiere a aquellos con concentraciones menores a $30 \mu \mathrm{g} \cdot \mathrm{mL}^{-1}$, extractos con moderado potencial aquellos que presenten un intervalo entre 30 y $100 \mu \mathrm{g} \cdot \mathrm{mL}^{-1}$ y extractos con bajo potencial antioxidante aquellos que presenten un $\mathrm{CI}_{50}$ por encima de $100 \mu \mathrm{g} \cdot \mathrm{mL}^{-1}$. No obstante, autores como Lee et al. (2008) describen que todo extracto que tenga efecto antioxidante con una concentración de $\mathrm{CI}_{50}$ menor a $10 \mathrm{mg} \cdot \mathrm{mL}^{-1}$ se puede considerar un antioxidante efectivo. 
En nuestros resultados, a pesar de que en los extractos de flor, por variedad o método de secado no se detectaron diferencias significativas en la actividad antioxidante, la actividad inhibidora del radical DPPH fue mayor que en plántula. En general podemos considerar que los valores obtenidos en este estudio tanto en plántula como en flor, evidencian una actividad antioxidante alta respecto a lo que se documenta en vainas de Vicia faba L. (Mateos-Aparicio et al., 2012), que mostraron un valor de $\mathrm{CI}_{50}$ de $400 \mu \mathrm{g} \cdot \mathrm{mL}^{-1}$.

\section{Correlación entre actividad antioxidante (CI50) frente al radical DPPH y el contenido de compuestos fenólicos totales y L-DOPA}

El contenido de CFT y de L-DOPA de los extractos de tejidos de plántula y flor de haba tuvieron correlaciones negativas estadísticamente significativas con la actividad antioxidante frente al radical DPPH $\left(\mathrm{CI}_{50}\right)$, con valores de correlación de -0.5049 y de -0.6041 respetivamente (Cuadro 4). Estos resultados sugieren que los CFT y L-DOPA de las plántulas y flores de haba juegan un papel significativo en los mecanismos antioxidantes con el radical DPPH. Estos hallazgos corroboran los resultados previos descritos por Mateos-Aparicio et al. (2012) que mencionan que en extractos de vainas de Vicia faba L., la fuerte actividad de atrapamiento de radicales contra el radical DPPH, estuvo relacionado primordialmente a los compuestos fenólicos. Además, se detectó una fuerte correlación $(\mathrm{r}=0.7917)$ entre el contenido de CFT con el contenido de LDOPA.

Cuadro 4. Análisis de correlación entre la actividad antioxidante frente al radical DPPH ( $\mathrm{CI}_{50}$ ) y el contenido de compuestos fenólicos totales (CFT) y de L-DOPA de plántulas y flores de Vicia faba L.

\begin{tabular}{|llll|}
\hline & DPPH (CI50) & CFT & L-DOPA \\
\hline DPPH $\left(\mathrm{CI}_{50}\right)$ & 1 & $-0.5049^{* * *}$ & $-0.6041^{* * *}$ \\
CFT & & 1 & $0.7917 * * *$ \\
L-DOPA & & \multicolumn{2}{c}{1} \\
\hline \multicolumn{2}{l}{$* * *=\mathrm{p}<0.001$ Altamente significativo. } \\
\hline
\end{tabular}

Al respecto Randhir y Shetty (2003) mostraron que plántulas de Vicia faba de 16 días tuvieron una disminución en la actividad antioxidante y conjuntamente un aumento en el contenido de LDOPA, lo que apunta a que en ese estado de desarrollo del haba, la mayoría de los fenólicos se 
canalizan hacia la producción de L-DOPA. Mientras que en el primer día de germinación de semillas de esta misma especie, se detectaron niveles altos de actividad antioxidante y también contenidos altos de L-DOPA, lo que sugiere que inicialmente los compuestos fenólicos son de naturaleza antioxidante y que solo una parte menor de ellos se convierten en lignina (Randhir y Shetty, 2004).

\section{Conclusiones}

En este estudio se evidenció la importancia de los métodos de secado en preservar los fitoquímicos y la actividad antioxidante de Vicia faba L. Los resultados mostraron la variación de los CFT, la L-DOPA y la actividad antioxidante frente al radical DPPH entre los métodos de secado por estufa y de liofilización en los tejidos de plántulas y flores de dos variedades de haba. Bajo las condiciones de estudio utilizadas en este trabajo, el secado por estufa de aire forzado a $38{ }^{\circ} \mathrm{C}$ tuvo la menor influencia sobre los contenidos de CFT, L-DOPA y la actividad antioxidante, por lo que este método podría ser aplicable debido a los niveles aceptables de CFT, L-DOPA y actividad antioxidante frente a los obtenidos por liofilización. La concentración de CFT y L-DOPA no se vio afectada por la variedad, pero si por el método de secado y la edad de las plántulas. La actividad antioxidante fue afectada por la variedad, método de secado y edad de las plántulas, pero en las flores no se detectaron diferencias por variedad o método de secado.

\section{$\underline{\text { Agradecimientos }}$}

Al MC Mario López Rodríguez del Instituto de Investigación y Capacitación Agropecuaria, Acuícola y Forestal del Estado de México (ICAMEX) y al MC Esteban Solórzano Vega de la Universidad Autónoma de Chapingo, por proporcionarnos las semillas que se utilizaron en este estudio. A la MC Cecilia García Osorio, por su apoyo en la detección de la L-DOPA en el equipo de HPLC. Al señor Daniel Mani por permitirnos utilizar su terreno para realizar el experimento en campo.

\section{Referencias}

Afshar, R. K., Chaichi, M. R., Jovini, M. A., Jahanzad, E., \& Hashemi, M. (2015). Accumulation of silymarin in milk thistle seeds under drought stress. Planta, 242(3), 539-543. doi: $10.1007 / \mathrm{s} 00425-015-2265-9$ 
Andrews, R. S \& Pridham, J. B. (1965). Structure of a dopa glucoside from Vicia faba. Revista Nature, 205(4977), 1213-1214. doi: 10.1038/2051213a0

Apaydin, H., Ertan, S., \& Özekmekçi, S. (2000). Broad bean (Vicia faba)—A natural source of L-dopa-Prolongs "on" periods in patients with Parkinson's disease who have "on-off" fluctuations. Movement disorders: official journal of the Movement Disorder Society, 15(1), 164-166. doi.org/10.1002/1531-8257

Asami, D. K., Hong, Y. J., Barrett, D. M., \& Mitchell, A. E. (2003). Comparison of the total phenolic and ascorbic acid content of freeze-dried and air-dried marionberry, strawberry, and corn grown using conventional, organic, and sustainable agricultural practices. Journal of Agricultural and Food Chemistry, 51(5), 1237-1241. doi: 10.1021/jf020635c

Brand-Williams, W., Cuvelier, M. E., \& Berset, C. L. W. T. (1995). Use of a free radical method to evaluate antioxidant activity. LWT-Food Science and Technology, 28(1), 25-30.

Cardador-Martínez, A., Maya-Ocaña, K., Ortiz-Moreno, A., Herrera-Cabrera, B. E., Dávila-Ortiz, G., Múzquiz, M., \& Jiménez-Martínez, C. (2012). Effect of Roasting and Boiling on the Content of Vicine, Convicine and L-3, 4-dihydroxyphenylalanine in Vicia faba L. Journal of Food Quality, 35(6), 419-428. doi: 10.1111/jfq.12006

Cenarruzabeitia, M. N., Soria, A. \& Larralde, J. (1978). The content of L-DOPA and its precursor L-tyrosine in Vicia faba grown in the north of Navarre. Anales de Edafologia $y$ Agrobiologia 37: 677-681

Chasquibol, N., Lengua, L., Delmás, I., Rivera, D., Bazán, D., Aguirre, R y Bravo, M. (2003). Alimentos funcionales o fitoquímicos, clasificación e importancia. Revista Peruana de Química e Ingeniería Química, 6(2), 9-20.

Chon, S. U. (2013). Total polyphenols and bioactivity of seeds and sprouts in several legumes. Current Pharmaceutical Design, 19(34), 6112-6124. doi: $10.2174 / 1381612811319340005$

Dahouda, M., Toleba, S. S., Youssao, A. K. I., Mama Ali, A. A., Dangou-Sapoho, R. K., Ahounou, S. G., \& Hornick, J. L. (2009). The effects of raw and processed Mucuna pruriens seed based diets on the growth parameters and meat characteristics of benin local guinea fowl (Numida meleagris, L.). International Journal of Poultry Science, 8(9), 882889. doi: 10.3923/ijps.2009.882.889 
Devi, K. V, Devi S. K., Shaikh, S. K. (2004). Plant based medications for Parkinsonism. Pharmatimes, 86, 41-45

Dewick, P. M. (2002). Medicinal natural products: a biosynthetic approach. Second Edition By P. M. Dewick (University of Nottingham). John Wiley \& Sons. UK. 507 p.

Drago Serrano, M. E., López López, M., \& Sainz Espuñes, T. D. R. (2006). Componentes bioactivos de alimentos funcionales de origen vegetal. Revista Mexicana de Ciencias Farmacéuticas, 37(4), 58-68.

Echeverría, G.C.L., \& Bressani, R. (2006). Effect of different cooking treatments of Mucuna beans on its L-DOPA content. Archivos Latinoamericanos de Nutrición, 56(2), 175-84.

Ehringer, H., \& Hornykiewicz, O. (1998). Distribution of noradrenaline and dopamine (3hydroxytyramine) in the human brain and their behavior in diseases of the extrapyramidal system. Parkinsonism and Related Disorders, 4(2), 53-57. doi: 10.1016/S13538020(98)00012-1

Etemadi, F., Hahsemi, M., Xing, B., \& Mashayekhi, H. (2014). Accumulation trend of L-DOPA in different parts of fava beans varieties. In Grand challenges, great solutions. Presentations of the ASA, CSSA, \& SSSA Annual Meetings, Long Beach, CA (pp. 2-5).

Etemadi, F., Hashemi, M., Randhir, R., ZandVakili, O., \& Ebadi, A. (2018a). Accumulation of LDOPA in various organs of faba bean and influence of drought, nitrogen stress, and processing methods on L-DOPA yield. The Crop Journal, 6(4), 426-434. doi:10.1016/j.cj.2017.12.001

Etemadi, F., Hashemi, M., Autio, W., Mangan, F., \& Zandvakili, O. (2018b). Accumulation and distribution trend of $L-D O P A$ in different parts of eight varieties of faba bean plant through its growth period. Journal of Crop Science, 6: 426-434.

Etemadi, F., Hashemi, M., Barker, A.V., Zandvakili, O.R., Liu, X. (2019) Agronomy, Nutritional Value, and Medicinal Application of Faba Bean (Vicia faba L.), Horticultural Plant Journal. doi:10.1016/j.hpj.2019.04.004

Foster, B. C., Arnason, J. T., \& Briggs, C. J. (2005). Natural health products and drug disposition. Annual Review of Pharmacology and Toxicology, 45, 203-226. doi: 10.1146/annurev.pharmtox.45.120403.095950 
Hu, J., Kwon, S. J., Park, J. J., Landry, E., Mattinson, D. S., \& Gang, D. R. (2015). LC-MS determination of L-DOPA concentration in the leaf and flower tissues of six faba bean (Vicia faba L.) lines with common and rare flower colors. Functional Foods in Health and Disease, 5(7), 243-250. doi: 10.31989/ffhd.v5i7.199

Hussian, G., \& Manyam, B. V. (1997). Mucuna pruriens proves more effective than L-DOPA in Parkinson's disease animal model. Phytotherapy Research: An International Journal Devoted to Medical and Scientific Research on Plants and Plant Products, 11(6), 419423.

Jankovic, J. (2002). Levodopa strengths and weaknesses. Neurology, 58(suppl 1), S19-S32.

Kedare, S. B., \& Singh, R. P. (2011). Genesis and development of DPPH method of antioxidant assay. Journal of food science and technology, 48(4), 412-422.

Kirakosyan, A., Kaufman, P. B., Duke, J. A., Warber, S., \& Bolling, S. (2004). The production of L-DOPA and isoflavones in seeds and seedlings of different cultivars of Vicia faba L. (fava bean). Evidence-Based Integrative Medicine, 1(2), 131-135.

Lang, A. \& Lozano, A. (1998) Parkinson's disease: First of two parts. The New England Journal of Medicine, 339, 1044-1053. doi:10.1056/NEJM199810083391506

Lee, Y.L., Jian, S.Y., Lian, P.Y., \& Mau, J.L. (2008). Antioxidant properties of extracts from a White mutant of the mushroom Hypsizigus marmoreus. Journal of Food Composition \& Analysis, 21, 116-124. doi: 10.1016/j.jfca.2007.09.005

Lewis, W. H., \& Elvin-Lewis, M. P. (2003). Medical botany: plants affecting human health. John Wiley $\quad \& \quad$ Sons. $\quad$ Consultado en https://books.google.com.mx/books?hl=es\&lr=\&id=ipQmSriMF9sC\&oi=fnd\&pg=PR9\& ots=4ob3k6DvzA\&sig=Alm3N2mh5X6ecWizATQmemDk3aI\#v=onepage \&q\&f=false

Lim, Y. Y., \& Murtijaya, J. (2007). Antioxidant properties of Phyllanthus amarus extracts as affected by different drying methods. LWT-Food Science and Technology, 40(9), 16641669. doi:10.1016/j.lwt.2006.12.013

Mateos-Aparicio, I., Redondo-Cuenca, A., \& Villanueva-Suárez, M. J. (2012). Broad bean and pea by-products as sources of fibre-rich ingredients: potential antioxidant activity measured in vitro. Journal of the Science of Food and Agriculture, 92(3), 697-703. doi: $10.1002 /$ jsfa.4633 
Mediani, A., Abas, F., Tan, C., \& Khatib, A. (2014). Effects of different drying methods and storage time on free radical scavenging activity and total phenolic content of Cosmos caudatus. Antioxidants, 3(2), 358-370. doi: 10.3390/antiox3020358

Menéndez-González, M., Castro-Santos, P., Suazo Galdames, I. C., \& Díaz-Peña, R. (2016) Farmacogenética en la Enfermedad de Parkinson: Influencia de Polimorfismos Genéticos Sobre los Efectos de la Terapia Dopaminérgica. Archivos de Medicina, 12 (3):9. doi: $10.3823 / 1308$

Nagatsu, T., \& Sawada, M. (2009). L-Dopa therapy for Parkinson's disease: past, present, and future. Parkinsonism \& related disorders, 15, S3-S8. doi.org/10.1016/S13538020(09)70004-5

Patil, S. A., Apine, O. A., Surwase, S. N., \& Jadhav, J. P. (2013). Biological sources of L-DOPA: An alternative approach. Advances in Parkinson's Disease, 2(03), 81. doi: 10.4236/apd.2013.23016

Pérez-Jiménez, J., Arranz, S., Tabernero, M., Díaz-Rubio, M. E., Serrano, J., Goñi, I., \& SauraCalixto, F. (2008). Updated methodology to determine antioxidant capacity in plant foods, oils and beverages: Extraction, measurement and expression of results. Food Research International, 41(3), 274-285. doi: 10.1016/j.foodres.2007.12.004

Petkovsek, M. M., Slatnar, A., Stampar, F., \& Veberic, R. (2010). The influence of organic/integrated production on the content of phenolic compounds in apple leaves and fruits in four different varieties over a 2-year period. Journal of the Science of Food and Agriculture, 90(14), 2366-2378. doi: 10.1002/jsfa.4093

Rabey, J.M., Vered, Y., Shabtai, H., Graff, E., \& Korczyn, A. D. (1992). Improvement of Parkinsonian features correlate with high plasma levodopa values after broad bean (Vicia faba) consumption. Journal of Neurosurgery and Psychiatry 55, 25-727. doi: 10.1136/jnnp.55.8.725

Ramos, A., Visozo, A., Piloto, J., García, A., Rodríguez, C.A., \& Rivero, R. (2003). Screening of antimutagenicity via antioxidant activity in Cuban medicinal plants. Journal of Ethnopharmacology, 87, 241-246. 
Randhir, R., \& Shetty, K. (2004). Microwave-induced stimulation of L-DOPA, phenolics and antioxidant activity in fava bean (Vicia faba) for Parkinson's diet. Process Biochemistry, 39(11), 1775-1784. doi: 10.1016/j.procbio.2003.08.006

SAS (Statistical Analysis Systems). (2002). SAS/STAT Users guide, version 9. SAS Institute Inc., North Carolina 14.

Shetty, P., Atallah, M. T., \& Shetty, K. (2001). Enhancement of total phenolic, L-DOPA and proline contents in germinating fava bean (Vicia faba) in response to bacterial elicitors. Food Biotechnology, 15(1), 47-67.

Shetty, K. (1997). Biotechnology to harness the benefits of dietary phenolics; focus on Lamiaceae. Asia Pacific Journal of Clinical Nutrition, 6(3), 162-171

Singleton, V. L., \& Rossi, J. A. (1965). Colorimetry of total phenolics with phosphomolybdicphosphotungstic acid reagents. American Journal of Enology and Viticulture, 16(3), 144158.

Siqueira-Soares, R. D. C., Soares, A. R., Parizotto, A. V., Ferrarese, M. D. L. L., \& FerrareseFilho, O. (2013). Root growth and enzymes related to the lignification of maize seedlings exposed to the allelochemical L-DOPA. The Scientific World Journal, 2013.

St Laurent, L., Livesey J., Arnason J. T. \& Bruneau A. (2000). Variation in L-dopa concentration in accessions of Mucuna pruriens (L.) DC var. utilis (Wall. ex Wight) Baker ex Burck. and in Mucuna brachycarpa Rech. In: Flores, B.M., Eilitta, M., Myhrman, R., Carew, L.B., Carsky, R.J. (ed.): Food and Feed from Mucuna: Current Uses and the Way Forward. Pp. 252-375. CIDICCO, CIEPCA and World Hunger Research Centre, Tegucigalpa.

Turco, I., Ferretti, G., \& Bacchetti, T. (2016) Review of the health benefits of Faba bean (Vicia faba L.) polyphenols. Journal of Food and Nutrition Research, 55 (4), 283-293

Vered, Y., Grosskopf, I., Palevitch, D., Harsat, A., Charach, G., Weintraub, M. S., \& Graff, E. (1997). The influence of Vicia faba (broad bean) seedlings on urinary sodium excretion. Planta Medica, 63(03), 237-240. 